\title{
Drug Treatment of Hyperlipidemia in Chinese Patients: Focus on the Use of Simvastatin and Ezetimibe Alone and in Combination
}

\author{
Sheng-Na Han ${ }^{1} \cdot$ Wei-Hong Yang ${ }^{1,2} \cdot$ Jian-Jian Yin ${ }^{1} \cdot$ Hai-Long Tao $^{3} \cdot$ Li-Rong Zhang $^{1} \mathbb{C}$
}

Published online: 4 February 2019

(c) The Author(s) 2019

\begin{abstract}
Elevated serum low-density lipoprotein cholesterol (LDL-C) is a major risk factor for coronary heart disease (CHD). Many guidelines recommend LDL-C as a primary treatment target, and statins represent the cornerstone of treatment for lipid management. Recently revised guidelines recommend even more intense management of LDL-C, especially in patients at moderate and high risk. However, LDL-C levels in the Chinese population differ from those in Western populations, and the benefits and safety of the maximum allowable dose of statins have yet to be determined. Furthermore, in practice, many patients do not achieve the increasingly stringent LDL-C goals. Consequently, alternative approaches to lipid management are required. Combination therapy with ezetimibe and a statin, which have complementary mechanisms of action, is more effective than statin monotherapies, even at high doses. Several clinical studies have consistently shown that combination therapy with ezetimibe and simvastatin lowers LDL-C more potently than statin monotherapies. Moreover, the safety and tolerability profile of the combination therapy appears to be similar to that of low-dose statin monotherapies. This review discusses the role of simvastatin in combination with ezetimibe in controlling dyslipidemia in Chinese patients, particularly the efficacy and safety of combination therapy in light of recently published clinical data.
\end{abstract}

\section{Key Points}

Simvastatin and ezetimibe combination therapy was more effective than statin monotherapy among Chinese patients with dyslipidemia.

Data suggest that combination therapy (simvastatin and ezetimibe) is more effective than statin monotherapy at lowering elevated serum low-density lipoprotein cholesterol levels.

Combination therapy neither compromises the safety and efficacy of the drugs nor increases the risk of health complications in treated patients.

Sheng-Na Han and Wei-Hong Yang have contributed equally to this work.

Li-Rong Zhang

zhanglirongzzu@126.com

1 Department of Pharmacology, School of Basic Medical Sciences, Zhengzhou University, 100 Science Road, Zhengzhou 450001, China

\section{Introduction to the Management of Lipid Disorders in Chinese Patients}

Coronary heart disease (CHD) is the second leading cause of cardiovascular death in the Chinese population [1]. In 2013, the mortality rate due to cardiovascular diseases (CVDs) was 295.63 per 100,000 individuals in rural areas and 261.99 per 100,000 in urban areas. In total, $44.6 \%$ of deaths in rural areas and $42.51 \%$ of deaths in urban areas were caused by CVDs, rates that are higher than for any other disease, including cancer [2]. Accumulating evidence shows that the incidence of CHD is steadily increasing in China [3]. Measures for the prevention and treatment of CHD in China should be enacted without delay.

Elevated serum cholesterol levels will result in an increase in CVD events of about 9.2 million between 2010 and 2030 in China [4]. The prevalence of dyslipidemia in Chinese adults was as high as $34.0 \%$ overall and $35.1 \%$ and

2 Department of Forensic Medicine, School of Basic Medical Sciences, Zhengzhou University, Zhengzhou, China

3 The First Affiliated Hospital of Zhengzhou University, Zhengzhou, China 
$26.3 \%$ in urban and rural areas, respectively [5]. Moreover, the role of low-density lipoprotein cholesterol (LDL-C) in the pathophysiology of CHD is acknowledged and wellunderstood, and the use of LDL-C-lowering medications has led to a significant reduction of cardiovascular risk in both primary and secondary prevention. Therefore, LDL-C is recommended as the primary treatment target for lipid management in many guidelines, such as the US National Cholesterol Education Program Adult Treatment Panel III (NCEP ATP III) guidelines [6], the 2012 Japan Atherosclerosis Society (JAS) guidelines [7], the 2013 American College of Cardiology (ACC)/American Heart Association (AHA) guidelines, the 2014/2015 National Lipid Association (NLA) recommendations for management of dyslipidemia in the context of evolving evidence [8], the 2016 European Society of Cardiology (ESC)/European Atherosclerosis Society (EAS) guidelines [9], and the 2016 Chinese guidelines for the management of adult dyslipidemia (revised edition) [10].

Since statins were introduced in the 1980s, they have emerged as one of the best-selling medication classes to date, with numerous trials demonstrating powerful efficacy in improving cardiovascular outcomes [11]. Statin therapy has become a cornerstone for the prevention and treatment of CHD and is generally safe and well-tolerated [9]. A number of large-scale clinical trials have demonstrated that statins substantially reduce cardiovascular morbidity and mortality as both primary and secondary prevention [12] and in high-risk patients [13]. The use of statins has revolutionized the management of CHD development risk, and more than 25 million individuals currently use statins worldwide [14]. However, despite the proven efficacy of statins in improving lipid profiles and reducing cardiovascular risk, a considerable proportion of statin-treated patients does not reach therapeutic LDL-C targets as defined by guidelines in China and other countries [15-17]. Many factors can influence the magnitude of response during lipid-altering therapy, including demographics (e.g., age, sex, race), metabolic disease (e.g., insulin resistance, diabetes mellitus, obesity, thyroid dysfunction), and treatment (e.g., statin type, dose, titration, combination therapy) [18].

However, how to use statins reasonably and effectively in the Chinese population has always been controversial. The ESC/EAS guidelines recommend the use of high-intensity statins (equivalent to the maximum allowable dose) at the beginning of treatment, but the benefits and safety of the maximum allowable dose of statins have not yet been determined in the Chinese population [9, 19]. The genetics, lifestyle, and diet of Chinese individuals differ from those in European and US populations, and $80 \%$ of Chinese residents have LDL-C levels $<130 \mathrm{mg} / \mathrm{dL}$, so fewer patients need high-dose statins to achieve a stronger lipid-lowering effect $[10,20]$. The HPS2-THRIVE study demonstrated that Chinese individuals had a much higher risk of myopathy with simvastatin $40 \mathrm{mg}$ monotherapy than did European subjects [21]. DYSIS (DYSlipidemia International Study), a large-scale cross-sectional survey of dyslipidemia management in patients receiving lipid-lowering agents in China, showed that the LDL-C control rates in high-risk and very high-risk populations were $54.8 \%$ and $39.7 \%$, respectively. It also showed that increasing the statin dose did not increase the medication compliance rate [22]. Therefore, most Chinese patients may not respond to the intensive statin dosage suggested by European guidelines. This highlights the need to explore an appropriate cholesterol-lowering therapy for high-risk patients in China. The CHILLAS trial did not show that high-intensity statins could provide more benefit to patients with acute coronary syndrome (ACS) in China [23], and the safety of high-intensity statins is a concern in the Chinese population. An increasing number of studies show that high-intensity statin treatment is accompanied by a higher risk of myopathy and elevated liver enzymes, which is also more prominent in the Chinese population. The study found no linear relationship between the efficacy and dosage of statins. The lipid-lowering efficacy of statins is characterized by initial lipid-lowering efficacy at the start of treatment. However, doubling the dose only decreased LDL-C by a further approximately $6 \%$, and the drug cost increased proportionally. Therefore, it is recommended [10] that moderate-strength statins be initially used, with the dosage appropriately adjusted according to the lipid-lowering effect and tolerance. If the cholesterol level is not to standard, a safe and effective lipid-lowering effect can be obtained by combining with other lipid-lowering drugs (class I recommendation, class B evidence) [10].

For high-risk patients who do not achieve adequate LDL-C lowering, use of a non-statin drug in combination with statins is recommended as an alternative therapeutic option. Ezetimibe was the first lipid-lowering agent found to inhibit the intestinal uptake of dietary and biliary cholesterol without affecting the absorption of fat-soluble nutrients [24]. When combined with statin therapy, the resulting complementary inhibition of cholesterol absorption and synthesis is more effective than statin monotherapy at reducing LDL-C, even at high statin doses. Furthermore, by combining ezetimibe with a statin, the requirement for statin dose titration can be reduced, thereby minimizing the risk of dose-related adverse events (e.g., myopathy, rhabdomyolysis, hepatotoxicity) [25]. Simvastatin with ezetimibe is available as a fixed-dose treatment that leads to a significant reduction in serum concentrations of the atherogenic LDL-C [26]. This review discusses the role of simvastatin in combination with ezetimibe in controlling dyslipidemia in Chinese patients in light of recently published clinical data. 


\section{Treatment Aims and Low-Density Lipoprotein Cholesterol (LDL-C) Targets}

LDL-C target levels are defined according to the patient's cardiovascular risk. In most people, atherosclerotic CVD (ASCVD) is the result of a number of risk factors, so all current guidelines on the prevention of CVD in clinical practice recommend the assessment of clinically manifest ASCVD and cardiovascular risk [9].

Several large clinical trials have shown that every $1 \mathrm{mmol} / \mathrm{L}$ reduction in LDL-C is associated with a $23 \%$ decrease in CVD risk [27]. According to the European guidelines, the presence of ASCVD, such as a history of acute myocardial infarction or stroke (i.e., secondary prevention), defines an LDL-C target of $<1.8 \mathrm{mmol} / \mathrm{L}$ ( $70 \mathrm{mg} /$ dL). In primary prevention for patients with type 2 diabetes mellitus (T2DM), or multiple risk factors leading to the estimation of high cardiovascular risk, an LDL-C level of $<2.6 \mathrm{mmol} / \mathrm{L}(100 \mathrm{mg} / \mathrm{dL})$ should be targeted. If these absolute treatment goals are not reached, LDL-C levels should be at least halved [28].

The 2013 AHA/ACC guidelines do not mention any absolute target LDL-C levels, instead recommending highintensity statin treatment to reduce LDL-C levels by $\geq 50 \%$ in patients with manifest ASCVD or high estimated risk and by $30-50 \%$ in patients with moderate risk. The new pooled cohort equations risk calculator qualifies a larger population for treatment with statins, which has been controversial $[29,30]$.

The 2016 revised Chinese guidelines suggest that both non-high-density lipoprotein cholesterol (HDL-C) and LDL-C be considered targets for lipid-altering therapy, and define therapeutic goals (Table 1) [10].

Studies in other countries have shown that the poor LDL-C control rate for high-risk patients treated in general practice is a global problem [31-33]. In South Africa, the control rate for very high-risk patients was reported to be only $26.5 \%$. Similarly, $67.2 \%$ of very high-risk patients in Lebanon and Jordan missed the goal $[34,35]$. Only $68 \%$ of patients in a high-risk cohort in Japan reached guideline-recommended LDL-C goals, and only $2 \%$ were treated with a statin, suggesting that statins are underutilized in high-risk Japanese populations [36]. Even in some developed regions, such as Europe and Canada, $45.3 \%$ of patients with diabetes were not at their LDL-C goal [37]. Despite the importance of achieving LDL-C targets, little improvement in LDL-C control has been realized for high-risk patients in China. DYSIS found that $62.5 \%$ of high-risk and very high-risk patients missed the LDL-C therapeutic goal in 2013 [22], which is only slightly better than the $74 \%$ reported for 2002 [38].

Failure to attain therapeutic LDL-C goals may be attributed to several factors in China [16]. First, patient and physician
Table 1 Treatment goals for non-high-density lipoprotein cholesterol and low-density lipoprotein cholesterol in atherosclerotic cardiovascular disease in China [10]

\begin{tabular}{lll}
\hline Risk category & LDL-C & Non-HDL-C \\
\hline Low or moderate & $<3.4 \mathrm{mmol} / \mathrm{L}$ & $<4.1 \mathrm{mmol} / \mathrm{L}$ \\
& $(130 \mathrm{mg} / \mathrm{dl})$ & $(160 \mathrm{mg} / \mathrm{dl})$ \\
High & $<2.6 \mathrm{mmol} / \mathrm{L}$ & $<3.4 \mathrm{mmol} / \mathrm{L}$ \\
& $(100 \mathrm{mg} / \mathrm{dl})$ & $(130 \mathrm{mg} / \mathrm{dl})$ \\
Very high & $<1.8 \mathrm{mmol} / \mathrm{L}$ & $<2.6 \mathrm{mmol} / \mathrm{L}$ \\
& $(70 \mathrm{mg} / \mathrm{dl})$ & $(100 \mathrm{mg} / \mathrm{dl})$ \\
\hline
\end{tabular}

$H D L-C$ high-density lipoprotein cholesterol, $L D L-C$ low-density lipoprotein cholesterol

adherence to lipid management remains poor, not least because hypercholesterolemia is an asymptomatic chronic condition, but also because some patients experience minor side effects with statin use. In addition, prescription rates for statins are $<50 \%$, even for high- or very high-risk patients [39]. Second, sex and obesity are strong risk factors for not reaching therapy goals, because women have a higher prevalence of hypercholesterolemia, and obese patients are often resistant to therapy and may require both combination drug therapy and lifestyle interventions. Finally, combination therapy as an alternative to high-dose statin monotherapy is often underused in clinical practice, primarily because of concerns about drug interactions and major adverse events. Cost is only a minor consideration in China, because most statins are covered by national medical insurance, and patients can receive full reimbursement for simvastatin. A survey of primary care physicians in 2010 [40] found that only $24 \%$ and $14 \%$ of participants could report the optimal LDL-C levels for patients with CHD and diabetes, respectively. Therefore, educational interventions to improve physicians' knowledge of lipid guidelines should be emphasized [17]. Creation of a national program to raise public awareness is also imperative.

\section{Cholesterol Metabolism and Pharmacology of Statins and Ezetimibe}

Cholesterol metabolism consists of a complex set of interactive input and output fluxes providing the large membrane-bound cholesterol pool with sufficient cholesterol molecules. Serum cholesterol is derived from the biosynthesis (endogenous pathway) and intestinal uptake (exogenous pathway) of dietary and biliary cholesterol [41]. Cholesterol is also catabolized to produce steroid hormones and bile acids [42]. Synthesis of cholesterol occurs in the cytoplasm and membrane of the endoplasmic reticulum of virtually all tissues in humans, including the liver, intestine, adrenal cortex, and reproductive tissues. Most of the circulating cholesterol is derived from 
internal manufacture rather than diet. The liver produces approximately $70 \%$ of total cholesterol (TC) in the body and when the liver can no longer produce it, blood cholesterol levels will subsequently fall [43]. The chemical pathway producing cholesterol in all cells begins with condensation of acetyl-CoA with acetoacetyl-CoA to form 3-hydroxy-3-methylglutaryl coenzyme A (HMG-CoA) in a reaction catalyzed by HMG-CoA synthase (Fig. 1). The HMG-CoA reductase (HMGCR) enzyme then converts HMG-CoA to mevalonate, which is the first rate-limiting step in this pathway [44]. This rate-limiting step is the conversion of HMG-CoA to CoA and mevalonate by a four-electron reductive deacylation process. After mevalonate is generated, multiple reactions follow to finally produce cholesterol. Statins are competitive, reversible inhibitors of HMGCR, the rate-limiting enzyme of cholesterol biosynthesis [45]. Statins, which modulate only endogenous cholesterol, inhibit biosynthesis of cholesterol, deplete intracellular pools, and enhance removal of plasma LDL-C [46], leading to significant reductions in serum LDL-C [47].

Statins exert lipid-lowering effects in two ways. The open ring portion of the chemical structure of inhibitors orally administered as active hydroxy acid forms (atorvastatin, fluvastatin, pitavastatin, pravastatin, and rosuvastatin) or prodrugs with a lactone ring (lovastatin and simvastatin) is similar to HMG-CoA [45]. The compounds therefore inhibit formation of mevalonate by competing with HMG-CoA, interrupting endogenous cholesterol synthesis and leading to a reduction in intracellular cholesterol synthesis. Simvastatin is administered as a lactone prodrug and is subsequently transformed to active metabolites. In vivo, lactone prodrugs are enzymatically hydrolyzed to their hydroxy acid pharmacophores in the liver to achieve pharmacological activity [48]. Alternately, reduced endogenous cholesterol synthesis in serum leads to increased LDL-C receptor expression on the surface of hepatocytes. Increased LDL-C receptor expression causes increased uptake of plasma LDL-C and consequently decreases plasma LDL-C levels [49].

The Niemann-Pick C1-like 1 (NPC1L1) sterol transporter, located at the intestinal wall brush-border membrane and canalicular membrane of the hepatocyte, plays a vital role in cholesterol absorption in the intestine and liver; hepatic NPC1L1 promotes the reabsorption of cholesterol from bile and decreases the loss of cholesterol from the liver [50]. Ezetimibe, a selective cholesterol absorption inhibitor, can effectively inhibit intestinal absorption of cholesterol from diet, along with bile and hepatic absorption, by binding to the NPC1L1 receptor, resulting in low cholesterol levels in vivo (Fig. 1) [51]. Ezetimibe monotherapy can reduce LDL-C levels by approximately $18 \%$, as well as triglyceride and apolipoprotein B (ApoB) levels by approximately 5\% and $15 \%$, respectively. Significant elevation of HDL-C has also been observed [52, 53].

The mechanisms by which NPC1L1 mediates cholesterol uptake and ezetimibe inhibits this process are only partially understood. NPC1L1 mediates cholesterol uptake via vesicular endocytosis, as cholesterol specifically promotes the incorporation of NPC1L1 into clathrin-coated vesicles [54]. Microfilaments and a complex of clathrin and its adapter protein AP2 mediate the endocytosis. At the same time, ezetimibe prevents NPC1L1 from incorporating into

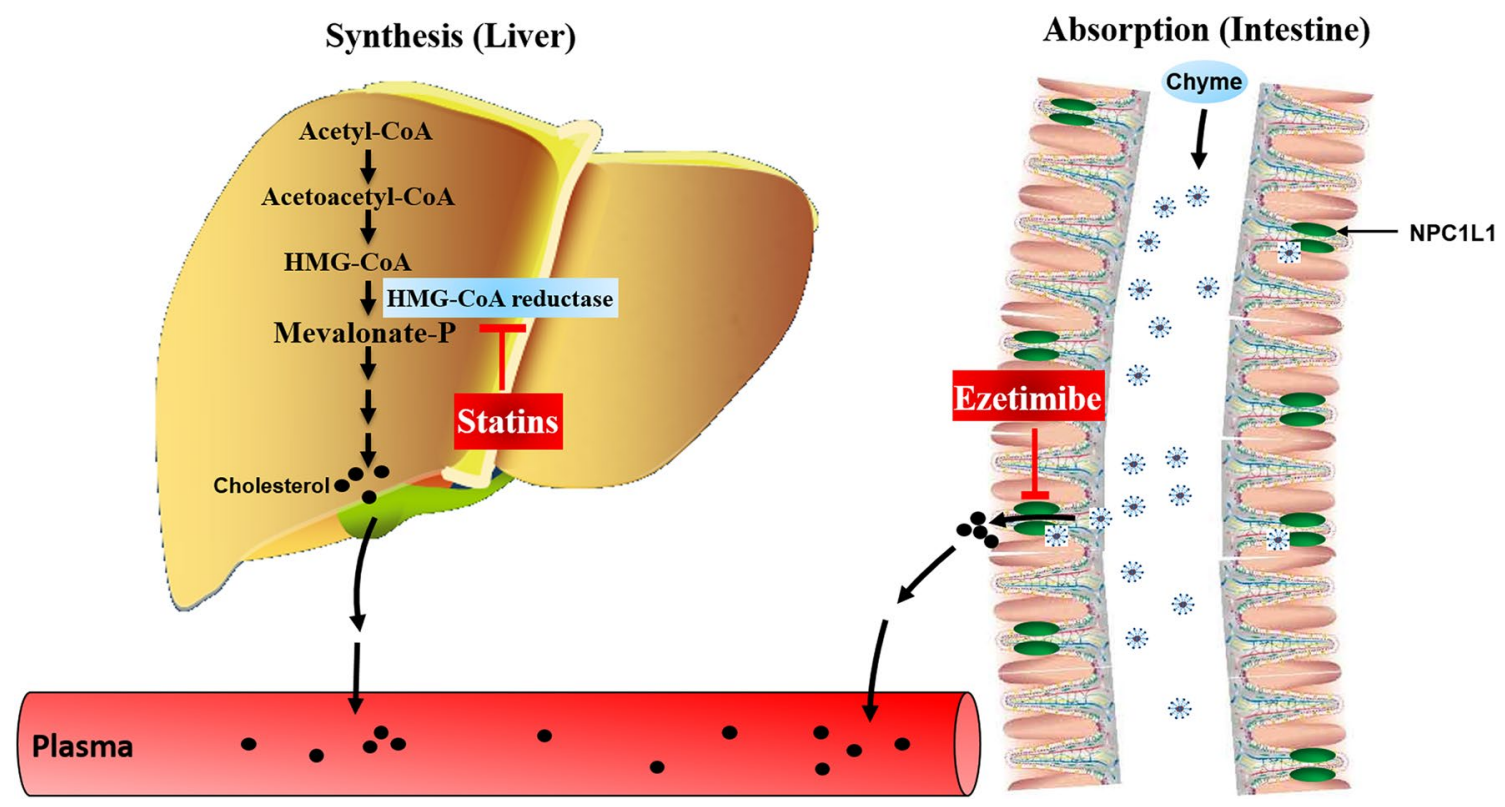

Fig. 1 Effects of statins and ezetimibe on cholesterol. HMG-CoA 3-hydroxy-3-methylglutaryl coenzyme A, NPC1L1 Niemann-Pick C1-like 1 
clathrin-coated vesicles and thus inhibits cholesterol uptake [54]. Recently, a mechanism for efficient free cholesterol absorption, the formation of cholesterol-enriched membrane microdomains positive for flotillins (NPC1L1 lipid raft proteins), was elucidated [55]. Ezetimibe may reduce plasma cholesterol by inhibiting NPC1L1 function in both intestine and liver, and hepatic NPC1L1 may protect the body from excessive biliary loss of cholesterol [56].

By inhibiting two independent pathways of plasma cholesterol generation - the exogenous pathway of cholesterol absorption from the gut (via ezetimibe) and the endogenous pathway of cholesterol biosynthesis in the liver (via simvastatin)—combination therapy may offer an efficacious strategy for the prevention and treatment of lipid disorders (Fig. 1). A number of clinical studies have consistently demonstrated that the simvastatin/ezetimibe combination provides more potent LDL-C lowering than statin monotherapy [18]. Thus, simvastatin/ezetimibe combination therapy reduces the need for statin dose titration and potentially decreases the adverse events associated with high-dose statin monotherapy. The SEAS (Simvastatin and Ezetimibe in Aortic Stenosis) study was a randomized, double-blind, placebocontrolled, multicenter study of a minimum 4 years' duration investigating the effect of lipid lowering with an ezetimibe/ simvastatin combination (10 mg/40 mg per day) in Western patients with asymptomatic aortic stenosis (AS). The study found that combination therapy with ezetimibe and a statin lowered LDL-C more than statins alone [57].

\section{Pharmacokinetics of Simvastatin Alone or in Combination with Ezetimibe in China}

Simvastatin is known to be absorbed in the intestine after administration as an inactive prodrug, and its absorption range is $80-85 \%$. Food intake does not affect its absorption. In a study of healthy Chinese subjects, the time to peak plasma concentration $\left(T_{\max }\right)$ was $0.7-3.0 \mathrm{~h}[58,59]$. In plasma, more than $95 \%$ of simvastatin is bound to plasma protein. Simvastatin undergoes phase I and phase II drug metabolism after oral administration. It can be readily converted into its active metabolite, simvastatin hydroxy acid, which serves as a potent competitive inhibitor of HMGCR by carboxylesterase or, without the enzyme, in the intestinal mucosa, plasma, and liver [60]. Simvastatin is then principally metabolized by phase I drug-metabolizing enzymes, cytochrome P450 (CYP)-3A4 and CYP3A5, in hepatic microsomes, where the former plays a major role [61]. Although simvastatin hydroxy acid can be metabolized by CYP3A4, CYP3A5, CYP2C8, and uridine 5'-diphosphoglucuronosyltransferase (UGT), CYP3A4 is the most important participant $[62,63]$. Moreover, simvastatin has a higher liver selectivity than simvastatin hydroxy acid
[64], promoting its metabolism. As simvastatin undergoes extensive first-pass metabolism in the liver, its bioavailability is not believed to exceed 5\% [65]. Simvastatin is glucuronidated via UGT $1 \mathrm{~A} 1$ and $1 \mathrm{~A} 3$ [61] and then excreted mainly via feces $(60 \%)$ and urine (13\%) [65]. Its elimination half-life $\left(t_{1 / 2}\right)$ in healthy Chinese subjects was reported to be 2.4-6.1 h [58].

Following oral administration, ezetimibe can also be rapidly absorbed in the liver and intestine and simultaneously extensively metabolized to its active ezetimibe-glucuronide form by glucuronidation of UGT [66]. $T_{\max }$ of total ezetimibe (free ezetimibe and ezetimibe-glucuronide) is $1-2 \mathrm{~h}$ post-administration [67]. Plasma protein binding rates of ezetimibe and ezetimibe-glucuronide conjugates are usually considered to be around $90 \%$ [68]. Ezetimibe and ezetimibe-glucuronide are excreted predominantly in the bile, while a small portion is excreted by the kidney. Because of enterohepatic recycling, the $t_{1 / 2}$ of ezetimibe and ezetimibe-glucuronide is approximately $22 \mathrm{~h}$ [68]. As ezetimibe is metabolized primarily by glucuronidation rather than oxidation of CYP, there are no significant interactions with other medications metabolized by the CYP pathway, such as simvastatin [69]. Thus, studies of the ezetimibe/ simvastatin combination have become a focus of physicians and researchers.

A recent study of the ezetimibe/simvastatin $10 \mathrm{mg} / 40 \mathrm{mg}$ combination in healthy Chinese subjects found that the pharmacokinetic parameters for ezetimibe and simvastatin were assessed by determining total ezetimibe, free ezetimibe, simvastatin, and simvastatin acid concentrations using a validated liquid chromatography-tandem mass spectrometry method. The results showed the following pharmacokinetic parameters of total ezetimibe and free ezetimibe: peak plasma concentration $\left(C_{\max }\right) 81.56 \pm 26.62$ and $9.40 \pm 6.17 \mathrm{ng} / \mathrm{mL}, T_{\max } 0.93 \pm 0.30$ and $1.25 \pm 1.27 \mathrm{~h}$, and $t_{1 / 2} 24.32 \pm 13.27$ and $18.90 \pm 9.66 \mathrm{~h}$, respectively. The pharmacokinetic parameters for simvastatin and simvastatin hydroxy acid following a single dose were $C_{\max } 11.92 \pm 5.50$ and $3.37 \pm 1.78 \mathrm{ng} / \mathrm{mL}, T_{\max } 0.98 \pm 0.28$ and $3.73 \pm 1.68 \mathrm{~h}$, and $t_{1 / 2} 4.19 \pm 1.81$ and $7.65 \pm 7.96 \mathrm{~h}$, respectively [70]. The pharmacokinetic characteristics of ezetimibe in healthy Chinese subjects were generally consistent with those in non-Chinese subjects [68]. However, in our study, the area under the concentration-time curve (AUC) and $C_{\max }$ were higher and $t_{1 / 2}$ was longer than results reported in 24 healthy Caucasian subjects [71]. These differences may be attributed to physical characteristics, and genetic polymorphisms may also be involved.

Pharmacokinetic studies suggest that the ezetimibe/simvastatin combination tablet is bioequivalent to the individual drug components administered together [72]. Coadministration of ezetimibe with simvastatin has no apparent effect on the pharmacokinetic characteristics of simvastatin [24]. 
In addition, ezetimibe does not appear to exert significant effects on plasma levels of other statins (atorvastatin, fluvastatin, lovastatin, pitavastatin, pravastatin, or rosuvastatin), fibric acid derivatives (gemfibrozil or fenofibrate), digoxin, glipizide, warfarin, or triphasic oral contraceptives (ethinyl estradiol and levonorgestrel) [68].

\subsection{Clinical Efficacy}

Simvastatin is widely used to treat elevated cholesterol levels, including TC, LDL-C, triglycerides, and ApoB, as monotherapy or in combination with other lipid-altering agents. Simvastatin monotherapy for 4 weeks has been shown to lower LDL-C by a mean of about $28 \%[73,74]$. However, not all subjects respond to statin treatment [75, 76]. A retrospective study of 2622 patients with hypercholesterolemia receiving statin monotherapy in China, Korea, Malaysia, Singapore, Taiwan, and Thailand [77] found that $48 \%$ of patients attained NCEP ATP III-recommended LDL-C goals. The attainment rate decreased as the risk category increased and was reported as $38 \%$ in the highrisk group, $62 \%$ in the moderate-risk group, and $81 \%$ in the low-risk group. Suboptimal cholesterol goal attainment has been observed in naturalistic clinical settings within other, non-Asian societies. For instance, in the REALITY-Europe study, approximately $40 \%$ of patients achieved their cholesterol targets, including 36\% of patients with CHD [78]. In the most recent EUROASPIRE II (European Action on Secondary Prevention by Intervention to Reduce Events) study, $41 \%$ of patients with CHD had TC "under control" $(5200 \mathrm{mg} / \mathrm{dL})$ [79]. While the overall goal attainment was surprisingly low (25\%), this phenomenon may be explained by the low proportion of lipid-lowering therapy $(<40 \%)$ prescribed in this population. It is known that each doubling of a statin's dose only results in an additional 5-7\% reduction in serum LDL-C levels but increases the risk of toxicity [80].

The efficacy of ezetimibe was evaluated in phase II [81] and phase III [82] trials in subjects with primary hypercholesterolemia who maintained an NCEP Step I or stricter diet [83]. Patients with primary hypercholesterolemia $(n=432$ [228 men, 204 women] mainly Caucasian, aged $18-75$ years) were included in this pooled analysis. Ezetimibe 5 or $10 \mathrm{mg}$ administered over 12 weeks significantly reduced LDL-C levels by $15.7 \%$ and $18.5 \%$, respectively ( $p<0.01$ vs. placebo), and significantly increased HDL-C levels by $2.9 \%$ and $3.5 \%$, respectively ( $p<0.05$ vs. placebo) $[81,82]$. Combination therapy with two hypolipidemic drugs may be required to meet target LDL-C levels recommended by the European Fifth Joint Task Force [84], NCEP ATP III [6], and Chinese guidelines for the management of dyslipidemias [10]. In a retrospective observational study in a Chinese population, patients were divided into three groups $(\mathrm{A}, \mathrm{B}$, and $\mathrm{C})$ to receive moderate-intensity statin, moderate ezetimibe-statin combination therapy, or intensive statin therapy, respectively. Moderate ezetimibe/statin combination therapy significantly reduced LDL-C levels to $69.1 \%$, whereas LDL-C levels in the intensive statin group and the moderate-intensity statin group decreased to $46.9 \%$ and $67.9 \%$, respectively [85]. A combination of drugs that act by different mechanisms can provide additive effects in LDL-C reduction [86]. As expected, trials of ezetimibe and statin combination therapy have revealed greater efficacy in LDL-C reduction than ezetimibe or statin monotherapy. A recent meta-analysis of over 21,000 subjects from 27 clinical trials randomizing adults to ezetimibe $10 \mathrm{mg}$ daily plus statin versus statin alone for 6-24 weeks found a significant $15.1 \%$ reduction in LDL-C with combination therapy compared with statin monotherapy. The study, in which the majority of participants were Caucasian (84\%), also demonstrated that combination therapy was more successful (32.1\%) than simvastatin monotherapy $(10.3 \%)$ in terms of subjects with CHD reaching the ATP III LDL-C goal of $<70 \mathrm{mg} / \mathrm{dL}$ [18].

In the BRAVO study of patients with hypercholesterolemia not reaching goal in Taiwan, $91 \%$ attained NCEP ATP III goals and experienced significant reductions in LDL-C, TC, and triglycerides, when treated with an ezetimibe/simvastatin combination [87]. Similarly, an openlabel, randomized controlled trial in Taiwanese patients with hypercholesterolemia demonstrated that the addition of ezetimibe to a moderate statin dose was significantly more effective at reducing LDL-C levels than doubling the dose of the statin [88]. In another study, Zhang et al. [89] found that TC and LDL-C levels decreased significantly compared with baseline levels after treatment with simvastatin $20 \mathrm{mg} /$ day alone for 4 weeks in patients with CHD. The total lipidlowering success rate in patients was $62 \%$. After 12 weeks, TC and LDL-C levels decreased significantly compared with levels after 4 weeks with a combination of simvastatin $20 \mathrm{mg} /$ day and ezetimibe $10 \mathrm{mg} /$ day, and the lipid-lowering success rate increased from zero to 59\% [89]. Rong et al. [90] found that LDL-C levels were better controlled with combined ezetimibe $10 \mathrm{mg} /$ day and simvastatin $20 \mathrm{mg} /$ day for 4 weeks, 8 weeks, and 12 weeks than with simvastatin alone. The combination therapy for 8 weeks and 12 weeks further lowered LDL-C and TC levels and improved goal attainment in Chinese patients with CHD and diabetes mellitus [90].

Cannon et al. [91] found that combining ezetimibe with simvastatin reduced LDL-C by an additional $23-24 \%$ in stable patients who had experienced an ACS. Moreover, the addition of ezetimibe to simvastatin therapy benefited more patients with diabetes than those without in the IMPROVEIT trial [92]. Nordestgaard et al. [92] reported that a statin/ ezetimibe combination could decrease LDL-C by $60-70 \%$ in patients with familial hypercholesterolemia $(\mathrm{FH})$. In view of the acceptable side effect profile and high compliance, 
the EAS recommends coadministration of ezetimibe as an add-on to statin therapy [93]. IMPROVE-IT, the first trial to demonstrate an incremental clinical benefit of adding a non-statin agent (ezetimibe) to statin therapy, found no significant differences in rates of adverse events between the combination therapy and monotherapy groups [94]. IMPROVE-IT also found that mutations in NPC1L1 were associated with reduced levels of LDL-C and a reduced risk of ASCVD [95]. Together, the trial data support the "LDL hypothesis" regarding reduction of cardiovascular events in patients with cardiovascular risk factors.

In Chinese patients with ACS and T2DM, combination therapy with ezetimibe and statins at doses used in stable CHD can provide better LDL-C-lowering effects and improve the control of LDL-C levels compared with statin monotherapy. Therefore, adding ezetimibe to moderate doses of statins is a more effective strategy for achieving significant additional LDL-C-lowering effects and for enabling more Chinese patients to achieve their LDL-C goals [95]. In China, sufficient training for primary care physicians regarding proper statin use and support for the combination of a statin and ezetimibe could promote LDL-C target attainment in primary care [16]; however, comprehensive data on the management of dyslipidemia and its treatment outcomes are limited.

\subsection{Safety and Tolerability}

The safety and tolerability of simvastatin alone or in combination with ezetimibe are of increasing importance to physicians and the public. Statins are well-tolerated by most patients; the most common side effects include dyspepsia, gastrointestinal discomfort, myalgia, headache, and central nervous system disturbances, though these are usually only of short duration [96]. Statins can also increase the risk of myopathy/rhabdomyolysis and are commonly associated with increased hepatic transaminase levels. However, these effects are infrequent [97]. Furthermore, Farmer and Torre-Amione [98] found that the increased hepatic transaminases induced by statins only occurred in approximately $1-3 \%$ of patients and was dose related, and that the majority of liver abnormalities occurred within the first 3 months of statin therapy and required monitoring. In addition, rhabdomyolysis, a rare, severe, and potentially lethal adverse effect, occurs in approximately $0.1 \%$ of patients with statin monotherapy; the incidence increases with coadministration of CYP3A4 and CYP3A5 inhibitors [98].

One pharmacokinetic study has shown that plasma exposure to simvastatin and its metabolites was significantly higher in Chinese, Malay, and Asian-Indian subjects than in Caucasian subjects living in the same environment [99]. However, evidence to date shows no increased rates of adverse events in Asian patients taking either lower or higher doses of statins [100, 101]. Simvastatin has also been reported to be effective in achieving the NCEP LDL-C target $(\leq 100 \mathrm{mg} / \mathrm{dL})$ and well-tolerated across the dose range of $20-80 \mathrm{mg} / \mathrm{dL}$ in Asian patients with CHD [92].

Furthermore, Rong et al. [90] demonstrated that ezetimibe/simvastatin combination therapy can have a favorable safety and tolerability profile in healthy Chinese subjects and in patients with CHD. Moreover, it has been demonstrated that ezetimibe/simvastatin therapy can lower LDL-C more effectively than simvastatin monotherapy in Chinese patients with an extremely high risk of CHD without adverse effects, indicating that tolerability and safety of the combination therapy is better [101].

Safety is another major argument against high-intensity statin use in the Chinese population. Mounting evidence suggests that a greater incidence of myopathy and elevated aminotransferase levels occurs in patients receiving highdose statins [102]. This excess risk may be particularly marked in Chinese patients. The HPS2-THRIVE study suggested that the absolute risk of myopathy and alanine transaminase elevation was much higher among participants receiving statins in China than among those in Europe (0.13 vs. $0.04 \%$ per year for myopathy, and 0.24 vs. $0.02 \%$ per year for elevated alanine transaminase) [21]. No safety data on high-intensity statin use in China are yet available. In short, there is currently no evidence to support high-intensity statin therapy for Chinese patients with CHD. In addition, available data have shown that higher doses are not associated with further risk reduction.

When ezetimibe is combined with simvastatin in Caucasian patients with primary hypercholesterolemia, the adverse event rate is generally similar to that observed with simvastatin alone [103]. In the IMPROVE-IT trial, discontinuation of study medication due to an adverse event occurred in $10.1 \%$ of patients taking simvastatin $40 \mathrm{mg}$ and in $10.6 \%$ of patients taking an ezetimibe/simvastatin combination [91]. However, data assessing the safety and tolerability of simvastatin in combination with ezetimibe in Chinese patients are limited.

\subsection{Patient-Focused Perspectives such as Quality of Life, Patient Satisfaction, Adherence, and Uptake}

Over many years of research and practice, statins have been shown to significantly reduce the incidence of cardiovascular events and mortality and improve patient satisfaction and quality of life. Simvastatin has been reported to be welltolerated at doses of $20-80 \mathrm{mg} / \mathrm{dL}$ in Asian patients with $\mathrm{CHD}$, and compliance with treatment was reported in 2001 to be approximately $80 \%$ [100]. Therefore, most patients can achieve satisfactory effects from therapy. For Chinese 
patients who do not attain target LDL-C levels with conventional-dose statin therapy, the efficacy of simvastatin/ ezetimibe combination treatment is significantly high, and the treatment costs less than atorvastatin monotherapy or atorvastatin/ezetimibe combination therapy.

According to Gao et al. [15], only $39.9 \%$ of patients with dyslipidemia in China receive lipid-lowering treatment, and only $25.8 \%$ of those surveyed achieved the NCEP ATP III LDL-C goal. The inability to achieve LDL-C goals has been attributed to a variety of causes, including improper dose titration, lack of follow-up, and-most importantly- poor adherence to treatment, in the form of irregular or interrupted intake and a high frequency of discontinuation. Therefore, good management strategies, appropriate therapeutic approaches, and good patient and physician adherence to recognized practice guidelines can play crucial roles in achieving favorable treatment outcomes.

\section{Conclusions}

Statins remain the cornerstone treatment in controlling lipid disorders in Chinese patients. More recent evidence-based data have shown that an intensive reduction in LDL-C levels, particularly in high-risk populations, is effective in reducing CHD risk. However, many patients do not achieve recommended LDL-C goals when treated in clinical practice. Alternative approaches to lipid management are required. Simvastatin and ezetimibe work via different mechanisms: the former inhibits the synthesis of cholesterol in the liver and the latter inhibits intestinal cholesterol absorption. Coadministration of these two complementary agents can further lower LDL-C levels in plasma while avoiding adverse reactions usually seen with high-dose statin therapy. Combination ezetimibe/simvastatin therapy is effective for achieving LDL-C goals as safely as the equivalent statin monotherapy. The advantages of this combination therapy have been supported by many clinical trials. Large multicenter cohort studies, including assessments of drug economics, are required to observe more comprehensive and precise results from ezetimibe/simvastatin combination therapy in the Chinese population.

\section{Acknowledgements None.}

\section{Compliance with Ethical Standards}

Funding This study was funded by National Natural Science Foundation of China (grant number 81670311).

Conflict of interest Sheng-Na Han, Wei-Hong Yang, Jian-Jian Yin, Hai-Long Tao and Li-Rong Zhang have no potential conflicts of interest that might be relevant to the contents of this manuscript.

Open Access This article is distributed under the terms of the Creative Commons Attribution-NonCommercial 4.0 International License (http://creativecommons.org/licenses/by-nc/4.0/), which permits any noncommercial use, distribution, and reproduction in any medium, provided you give appropriate credit to the original author(s) and the source, provide a link to the Creative Commons license, and indicate if changes were made.

\section{References}

1. Zhang XH, Lu ZL, Liu L. Coronary heart disease in China. Heart. 2008;94(9):1126-31. https://doi.org/10.1136/hrt.2007.132423.

2. Chen WW, Gao RL, Liu LS, Zhu ML, Wang W, Wang YJ, et al. China cardiovascular diseases report 2015: a summary. J Geriatr Cardiol. 2017;14(1):1-10. https://doi.org/10.11909/j.i ssn.1671-5411.2017.01.012.

3. Wu Z, Yao C, Zhao D, Wu G, Wang W, Liu J, et al. Sino-MONICA project: a collaborative study on trends and determinants in cardiovascular diseases in China, Part i: morbidity and mortality monitoring. Circulation. 2001;103(3):462-8.

4. Moran A, Gu D, Zhao D, Coxson P, Wang YC, Chen CS, et al. Future cardiovascular disease in china: markov model and risk factor scenario projections from the coronary heart disease policy model-china. Circ Cardiovasc Qual Outcomes. 2010;3(3):24352. https://doi.org/10.1161/CIRCOUTCOMES.109.910711.

5. Pan L, Yang ZH, Wu Y, Yin RX, Liao YH, Wang JW, et al. The prevalence, awareness, treatment and control of dyslipidemia among adults in China. Atherosclerosis. 2016;248:2-9. https:// doi.org/10.1016/j.atherosclerosis.2016.02.006.

6. Grundy SM, Becker D, Clark LT, Cooper RS, Denke MA, Howard WJ, et al. Third Report of the National Cholesterol Education Program (NCEP) expert panel on detection, evaluation, and treatment of high blood cholesterol in adults (Adult Treatment Panel III) final report. Circulation. 2002;106(25):3143-421.

7. Teramoto T, Sasaki J, Ishibashi S, Birou S, Daida H, Dohi S, et al. Diagnosis of Atherosclerosis Executive Summary of the Japan Atherosclerosis Society (JAS) Guidelines for the Diagnosis and Prevention of Atherosclerotic Cardiovascular Diseases in Japan-2012 Version. J Atherosclerosis Thromb. 2014;21(4):296303. https://doi.org/10.5551/jat.19737.

8. Waite LH, Phan YL, Spinler SA. What's next for dyslipidemia management? The 2013 ACC/AHA Guidelines, the NLA recommendations, and beyond. J Am Pharm Assoc (2003). 2016;56(3):284-92. https://doi.org/10.1016/j.japh.2015.12.017.

9. Authors/Task Force M:, Catapano AL, Graham I, De Backer G, Wiklund O, Chapman MJ et al. ESC/EAS guidelines for the management of dyslipidaemias: the task force for the management of dyslipidaemias of the European Society of Cardiology (ESC) and European Atherosclerosis Society (EAS) Developed with the special contribution of the European Assocciation for Cardiovascular Prevention and Rehabilitation (EACPR). Atherosclerosis. 2016;2016(253):281-344. https://doi.org/10.1016/j.atheroscle rosis.2016.08.018.

10. Zhu JR, Gao RL, Zhao SP, Lu GP, Zhao D, Li JJ. Guidelines for management of adult dyslipidemia in China. Chin Circ J. 2016;31(10):937-63. https://doi.org/10.3969/j. issn.1000-3614.2016.10.001

11. Kapur NK, Musunuru K. Clinical efficacy and safety of statins in managing cardiovascular risk. Vasc Health Risk Manag. 2008;4(2):341-53.

12. Fulcher J, O'Connell R, Voysey M, Emberson J, Blackwell L, Mihaylova B, et al. Efficacy and safety of LDL-lowering therapy among men and women: meta-analysis of individual data from 174000 participants in 27 randomised trials. Lancet. 
2015;385(9976):1397-405. https://doi.org/10.1016/S0140 -6736(14)61368-4.

13. Agouridis AP, Elisaf MS, Nair DR, Mikhailidis DP. All for statins and statins for all; An update. Curr Pharm Des. 2016;22(1):1827. https://doi.org/10.2174/1381612822666151109111511.

14. Khang AR, Song YS, Kim KM, Moon JH, Lim S, Park KS, et al. Comparison of different statin therapy to change low-density lipoprotein cholesterol and high-density lipoprotein cholesterol level in Korean patients with and without diabetes. J Clin Lipidol. 2016;10(3):528-37. https://doi.org/10.1016/j.jacl.2015.12.013.

15. Gao F, Zhou YJ, Hu DY, Zhao YX, Liu YY, Wang ZJ, et al. Contemporary management and attainment of cholesterol targets for patients with dyslipidemia in China. PLoS One. 2013;8(4):e47681. https://doi.org/10.1371/journal.pone.00476 81.

16. Lin X, Xu R, Fang L, Chen W, Hu D, Zhao S, et al. Factors influencing low-density lipoprotein cholesterol target achievement in primary care-results from DYSIS China. Int J Cardiol. 2016;222:51-6. https://doi.org/10.1016/j.ijcard.2016.07.047.

17. Gitt AK, Lautsch D, Ferrieres J, Kastelein J, Drexel H, Horack $\mathrm{M}$, et al. Low-density lipoprotein cholesterol in a global cohort of 57,885 statin-treated patients. Atherosclerosis. 2016;255:200-9. https://doi.org/10.1016/j.atherosclerosis.2016.09.004.

18. Morrone D, Weintraub WS, Toth PP, Hanson ME, Lowe RS, Lin $\mathrm{J}$, et al. Lipid-altering efficacy of ezetimibe plus statin and statin monotherapy and identification of factors associated with treatment response: a pooled analysis of over 21,000 subjects from 27 clinical trials. Atherosclerosis. 2012;223(2):251-61. https:// doi.org/10.1016/j.atherosclerosis.2012.02.016.

19. Dai W, Huang XS, Zhao SP. No evidence to support highintensity statin in Chinese patients with coronary heart disease. Int J Cardiol. 2016;1(204):57-8. https://doi.org/10.1016/j.ijcar d.2015.11.164.

20. Sun D, Zhou BY, Li S, et al. Genetic basis of index patients with familial hypercholesterolemiain Chinese population: mutation spectrum and genotype-phenotype correlation. Lipids Health Dis. 2018;17(1):252. https://doi.org/10.1186/s12944-018-0900-8.

21. Group HTC. HPS2-THRIVE randomized placebo-controlled trial in 25673 high-risk patients of ER niacin/laropiprant: trial design, pre-specified muscle and liver outcomes, and reasons for stopping study treatment. Eur Heart J. 2013;34(17):1279-91. https ://doi.org/10.1093/eurheartj/eht055.

22. Zhao S, Wang Y, Mu Y, Yu B, Ye P, Yan X, et al. Prevalence of dyslipidaemia in patients treated with lipid-lowering agents in China: results of the DYSlipidemia International Study (DYSIS). Atherosclerosis. 2014;235(2):463-9. https://doi.org/10.1016/j. atherosclerosis.2014.05.916.

23. Zhao SP, Yu BL, Peng DQ, Huo Y. The effect of moderate-dose versus double-dose statins on patients with acute coronarysyndrome in China: results of the CHILLAS trail. Atherosclerosis. 2014;233(2):707-12. https://doi.org/10.1016/j.atherosclerosis .2013.12.003.

24. Kosoglou T, Meyer I, Veltri EP, Statkevich P, Yang B, Zhu Y, et al. Pharmacodynamic interaction between the new selective cholesterol absorption inhibitor ezetimibe and simvastatin. $\mathrm{Br}$ J Clin Pharmacol. 2002;54(3):309-19. https://doi.org/10.104 6/j.1365-2125.2002.01633.x.

25. Thompson PD, Clarkson P, Karas RH. Statin-associated myopathy. JAMA. 2003;289(13):1681-90. https://doi.org/10.1001/ jama.289.13.1681.

26. Agouridis AP, Filippatos TD, Katsiki N. Current lipid-modifying agents. Exp Opin Pharmacother. 2015;16(7):1117-8. https ://doi.org/10.1517/14656566.2015.1035013.

27. Silverman MG, Ference BA, Im K, Wiviott SD, Giugliano RP, Grundy SM, et al. Association between lowering LDL-C and cardiovascular risk reduction among different therapeutic interventions a systematic review and meta-analysis. JAMA. 2016;316(12):1289-97. https://doi.org/10.1001/ jama.2016.13985.

28. Krahenbuhl S, Pavik-Mezzour I, von Eckardstein A. Unmet needs in LDL-C lowering: when Statins Won't Do! Drugs. 2016;76(12):1175-90. https://doi.org/10.1007/s4026 5-016-0613-0.

29. Kavousi M, Leening MJ, Nanchen D, Greenland P, Graham IM, Steyerberg EW, et al. Comparison of application of the ACC/AHA guidelines, Adult Treatment Panel III guidelines, and European Society of Cardiology guidelines for cardiovascular disease prevention in a European cohort. JAMA. 2014;311(14):1416-23. https://doi.org/10.1001/ jama.2014.2632.

30. Colantonio LD, Baber U, Banach M, Tanner RM, Warnock DG, Gutierrez OM, et al. Contrasting cholesterol management guidelines for adults with CKD. J Am Soc Nephrol. 2015;26(5):117380. https://doi.org/10.1681/ASN.2014040400.

31. Jameson K, Amber V, D’Oca K, Mills D, Giles A, Ambegaonkar B. Impact of lipid-lowering therapy on the prevalence of dyslipidaemia in patients at high-risk of cardiovascular events in UK primary care-a retrospective database study. Int J Clin Pract. 2013;67(12):1228-37. https://doi.org/10.1111/ijcp.12238.

32. Unniachan S, Bash LD, Khovidhunkit W, Sri RZT, Vicaldo E, Recto C, et al. Prevalence of lipid abnormalities and attainment of normal lipid levels among patients with dyslipidaemia: a pooled analysis of observational studies from five Asian countries. Int J Clin Pract. 2014;68(8):1010-9. https://doi. org/10.1111/ijcp.12407.

33. Devroey D, Radermecker RP, Van der Schueren BJ, Torbeyns B, Jaken RJ. Prevalence of persistent lipid abnormalities in statintreated patients: belgian results of the Dyslipidaemia International Study (DYSIS). Int J Clin Pract. 2014;68(2):180-7. https ://doi.org/10.1111/ijcp.12315.

34. Raal FJ, Blom DJ, Naidoo S, Bramlage P, Brudi P. Prevalence of dyslipidaemia in statin-treated patients in South Africa: results of the DYSlipidaemia International Study (DYSIS). Cardiovasc J Afr. 2013;24(8):330-8. https://doi.org/10.5830/CVJA-2013-071.

35. Azar ST, Hantash HA, Jambart S, El-Zaheri MM, Rachoin R, Chalfoun A, et al. Factors influencing dyslipidemia in statin-treated patients in Lebanon and Jordan: results of the Dyslipidemia International Study. Vasc Health Risk Manag. 2014;10:225-35. https://doi.org/10.2147/VHRM.S57194.

36. Teramoto T, Uno K, Miyoshi I, Khan I, Gorcyca K, Sanchez RJ, et al. Low-density lipoprotein cholesterol levels and lipid-modifying therapy prescription patterns in the real world: an analysis of more than 33,000 high cardiovascular risk patients in Japan. Atherosclerosis. 2016;251:248-54. https://doi.org/10.1016/j. atherosclerosis.2016.07.001.

37. Gitt AK, Drexel H, Feely J, Ferrieres J, Gonzalez-Juanatey JR, Thomsen KK, et al. Persistent lipid abnormalities in statin-treated patients and predictors of LDL-cholesterol goal achievement in clinical practice in Europe and Canada. Eur J Prev Cardiol. 2012;19(2):221-30. https://doi.org/10.1177/1741826711400545.

38. Wu YF. A multi-center study of current status on clinical control of hypercholesterolemia in China: success rate and related factors. Chin J Cardiol. 2002;30(2):109-14. https://doi. org/10.3760/j:issn:0253-3758.2002.02.014

39. Park JE, Chiang CE, Munawar M, Pham GK, Sukonthasarn A, Aquino AR, et al. Lipid-lowering treatment in hypercholesterolaemic patients: the CEPHEUS Pan-Asian survey. Eur J Prev Cardiol. 2012;19(4):781-94. https://doi.org/10.1177/17418 26710397100.

40. Guan F, Xie J, Wang GL, Wang JH, Wang JS, Yu JM, et al. Community-wide survey of physicians' knowledge of cholesterol management. Chin Med J Engl. 2010;123(7):884-9. 
41. Beisiegel U. Lipoprotein metabolism. Eur Heart J. 1998;19(Suppl A):A20-3.

42. Stellaard F, von Bergmann K, Sudhop T, Lutjohann D. The value of surrogate markers to monitor cholesterol absorption, synthesis and bioconversion to bile acids under lipid lowering therapies. J Steroid Biochem Mol Biol. 2017;169:111-22. https ://doi.org/10.1016/j.jsbmb.2016.03.030.

43. Egom EE, Hafeez H. Biochemistry of statins. Adv Clin Chem. 2016;73:127-68. https://doi.org/10.1016/bs.acc.2015.10.005.

44. Stancu C, Sima A. Statins: mechanism of action and effects. J Cell Mol Med. 2001;5(4):378-87.

45. Shitara Y, Sugiyama Y. Pharmacokinetic and pharmacodynamic alterations of 3-hydroxy-3-methylglutaryl coenzyme A (HMG$\mathrm{CoA}$ ) reductase inhibitors: drug-drug interactions and interindividual differences in transporter and metabolic enzyme functions. Pharmacol Ther. 2006;112(1):71-105. https://doi.org/10.1016/j. pharmthera.2006.03.003.

46. Vaughan CJ, Murphy MB, Buckley BM. Statins do more than just lower cholesterol. The Lancet. 1996;348(9034):1079-82. https:// doi.org/10.1016/s0140-6736(96)05190-2.

47. Malinowski JM. Atorvastatin: a hydroxymethylglutarylcoenzyme A reductase inhibitor. Am J Health Syst Pharm. 1998;55(21):2253-67.

48. Corsini A, Maggi FM, Catapano AL. Pharmacology of competitive inhibitors of HMG-CoA reductase. Pharmacol Res. 1995;31(1):9-27.

49. Lennernas H, Fager G. Pharmacodynamics and pharmacokinetics of the HMG-CoA reductase inhibitors. Similarities and differences. Clin Pharmacokinet. 1997;32(5):403-25. https://doi. org/10.2165/00003088-199732050-00005.

50. Altmann SW, Davis HR, Zhu LJ, Yao XR, Hoos LM, Tetzloff $\mathrm{G}$, et al. Niemann-Pick C1 like 1 protein is critical for intestinal cholesterol absorption. Science. 2004;303(5661):1201-4. https ://doi.org/10.1126/science.1093131.

51. Chang TY, Chang C. Ezetimibe blocks internalization of the NPC1L1/cholesterol complex. Cell Metab. 2008;7(6):469-71. https://doi.org/10.1016/j.cmet.2008.05.001.

52. Knopp RH, Gitter H, Truitt T, Bays H, Manion CV, Lipka LJ, et al. Effects of ezetimibe, a new cholesterol absorption inhibitor, on plasma lipids in patients with primary hypercholesterolemia. Eur Heart J. 2003;24(8):729-41.

53. Dujovne CA, Ettinger MP, McNeer JF, Lipka LJ, LeBeaut AP, Suresh R, et al. Efficacy and safety of a potent new selective cholesterol absorption inhibitor, ezetimibe, in patients with primary hypercholesterolemia. Am J Cardiol. 2002;90(10):1092-7.

54. Ge L, Wang J, Qi W, Miao HH, Cao J, Qu YX, et al. The cholesterol absorption inhibitor ezetimibe acts by blocking the sterolinduced internalization of NPC1L1. Cell Metab. 2008;7(6):50819. https://doi.org/10.1016/j.cmet.2008.04.001.

55. Ge LA, Qi W, Wang LJ, Miao HH, Qu YX, Li BL, et al. Flotillins play an essential role in Niemann-Pick C1-like 1-mediated cholesterol uptake. Proc Natl Acad Sci USA. 2011;108(2):551-6. https://doi.org/10.1073/pnas.1014434108.

56. Temel RE, Tang W, Ma Y, Rudel LL, Willingham MC, Ioannou YA, et al. Hepatic Niemann-Pick C1-like 1 regulates biliary cholesterol concentration and is a target of ezetimibe. J Clin Invest. 2007;117(7):1968-78. https://doi.org/10.1172/JCI30060.

57. Rossebo AB, Pedersen TR, Allen C, Boman K, Chambers J, Egstrup K, et al. Design and baseline characteristics of the simvastatin and ezetimibe in aortic stenosis (SEAS) study. Am J Cardiol. 2007;99(7):970-3. https://doi.org/10.1016/j.amjca rd.2006.10.064

58. Zhang P, Lian JP. Relative bioavailability study of simvastatin tablets in healthy volunteers. Mod J Integr Trade Chin Western Med. 2013;22(9):984-6. https://doi.org/10.3969/j. issn.1008-8849.2013.09.035
59. Zhou Q, Ruan ZR, Jiang B, Yuan H, Zeng S. Simvastatin pharmacokinetics in healthy Chinese subjects and its relations with CYP2C9, CYP3A5, ABCB1, ABCG2 and SLCO1B1 polymorphisms. Pharmazie. 2013;68(2):124-8.

60. Pasanen MK, Neuvonen M, Neuvonen PJ, Niemi M. SLCO1B1 polymorphism markedly affects the pharmacokinetics of simvastatin acid. Pharmacogenet Genom. 2006;16(12):873-9. https ://doi.org/10.1097/01.fpc.0000230416.82349.90.

61. Prueksaritanont T, Gorham LM, Ma B, Liu L, Yu X, Zhao JJ, et al. In vitro metabolism of simvastatin in humans [SBT] identification of metabolizing enzymes and effect of the drug on hepatic P450s. Drug Metab Dispos. 1997;25(10):1191-9.

62. Prueksaritanont T, Ma B, Yu N. The human hepatic metabolism of simvastatin hydroxy acid is mediated primarily by CYP3A, and not CYP2D6. Br J Clin Pharmacol. 2003;56(1):120-4.

63. Prueksaritanont T, Subramanian R, Fang X, Ma B, Qiu Y, Lin $\mathrm{JH}$, et al. Glucuronidation of statins in animals and humans: a novel mechanism of statin lactonization. Drug Metab Dispos. 2002;30(5):505-12.

64. Koga T, Fukuda K, Shimada Y, Fukami M, Koike H, Tsujita $\mathrm{Y}$. Tissue selectivity of pravastatin sodium, lovastatin and simvastatin. The relationship between inhibition of de novo sterol synthesis and active drug concentrations in the liver, spleen and testis in rat. Eur J Biochem. 1992;209(1):315-9.

65. Todd PA, Goa KL. Simvastatin. A review of its pharmacological properties and therapeutic potential in hypercholesterolaemia. Drugs. 1990;40(4):583-607.

66. Ghosal A, Hapangama N, Yuan Y, Achanfuo-Yeboah J, Iannucci $\mathrm{R}$, Chowdhury $\mathrm{S}$, et al. Identification of human UDP-glucuronosyltransferase enzyme(s) responsible for the glucuronidation of ezetimibe (Zetia). Drug Metab Dispos. 2004;32(3):314-20. https ://doi.org/10.1124/dmd.32.3.314.

67. Ezetimibe BH. Expert Opin Investig Drug. 2002;11(11):1587604. https://doi.org/10.1517/13543784.11.11.1587.

68. Kosoglou T, Statkevich P, Johnson-Levonas AO, Paolini JF, Bergman AJ, Alton KB. Ezetimibe: a review of its metabolism, pharmacokinetics and drug interactions. Clin Pharmacokinet. 2005;44(5):467-94.

69. Jeu L, Cheng JW. Pharmacology and therapeutics of ezetimibe (SCH 58235), a cholesterol-absorption inhibitor. Clin Ther. 2003;25(9):2352-87.

70. Chu NN, Chen WL, Xu HR, Li XN. Pharmacokinetics and safety of ezetimibe/simvastatin combination tablet: an open-label, single-dose study in healthy Chinese subjects. Clin Drug Investig. 2012;32(12):791-8. https://doi.org/10.1007/s40261-012-0013-5.

71. Najib NM, Idkaidek N, Adel A, Admour I, Astigarraga RE, Nucci GD, et al. Pharmacokinetics and bioequivalence evaluation of two simvastatin $40 \mathrm{mg}$ tablets (Simvast and Zocor) in healthy human volunteers. Biopharm Drug Dispos. 2003;24(5):183-9. https://doi.org/10.1002/bdd.347.

72. Migoya EM, Bergman A, Hreniuk D, Matthews N, Yi B, Roadcap B, et al. Bioequivalence of an ezetimibe/simvastatin combination tablet and coadministration of ezetimibe and simvastatin as separate tablets in healthy subjects. Int J Clin Pharmacol Ther. 2006;44(2):83-92.

73. Gao Y, Zhang LR, Fu Q. CYP3A4*1G polymorphism is associated with lipid-lowering efficacy of atorvastatin but not of simvastatin. Eur J Clin Pharmacol. 2008;64(9):877-82. https://doi. org/10.1007/s00228-008-0502-X.

74. Li YP, Zhang LR, Jia M, Hu XJ. CYP3AP1*3 allele is associated with lipid-lowering efficacy of simvastatin and atorvastatin in Chinese women. J Clin Pharmacol. 2011;51(2):181-8. https:// doi.org/10.1177/0091270010370589.

75. Yu BL, Zhao SP, Peng DQ, Huo Y, Hu L. A comparison of nonHDL and LDL cholesterol goal attainment in the CHILLAS trial. 
Int J Cardiol. 2013;168(4):4340-2. https://doi.org/10.1016/j.ijcar d.2013.05.080.

76. Lee KK, Lee VW, Chan WK, Lee BS, Chong AC, Wong JC, et al. Cholesterol goal attainment in patients with coronary heart disease and elevated coronary risk: results of the Hong Kong hospital audit study. Value Health. 2008;11(Suppl 1):S91-8. https://doi.org/10.1111/j.1524-4733.2008.00372.x.

77. Kim HS, Wu YF, Lin SJ, Deerochanawong CC, Zarnbahari R, Zhao LC, et al. Current status of cholesterol goal attainment after statin therapy among patients with hypercholesterolemia in Asian countries and region: the Return on Expenditure Achieved for Lipid Therapy in Asia (REALITY-Asia) study. Curr Med Res Opin. 2008;24(7):1951-63. https://doi.org/10.1185/0300799080 2138731.

78. Van Ganse E, Laforest L, Alemao E, Davies G, Gutkin S, Yin D. Lipid-modifying therapy and attainment of cholesterol goals in Europe: the Return on Expenditure Achieved for Lipid Therapy (REALITY) study. Curr Med Res Opin. 2005;21(9):1389-99. https://doi.org/10.1185/030079905X59139.

79. Euroaspire I, Group II, European Action on Secondary Prevention by Intervention to Reduce E, Reduce E. Clinical reality of coronary prevention guidelines: a comparison of EUROASPIRE I and II in nine countries. EUROASPIRE I and II Group. European Action on Secondary Prevention by Intervention to Reduce Events. The Lancet. 2001;357(9261):995-1001.

80. Nicholls SJ, Brandrup-Wognsen G, Palmer M, Barter PJ. Meta-analysis of comparative efficacy of increasing dose of Atorvastatin versus Rosuvastatin versus Simvastatin on lowering levels of atherogenic lipids (from VOYAGER). Am J Cardiol. 2010;105(1):69-76. https://doi.org/10.1016/j.amjca rd.2009.08.651.

81. Bays HE, Moore PB, Drehobl MA, Rosenblatt S, Toth PD, Dujovne CA, et al. Effectiveness and tolerability of ezetimibe in patients with primary hypercholesterolemia: pooled analysis of two phase II studies. Clin Ther. 2001;23(8):1209-30.

82. Knopp RH, Gitter H, Truitt T, Lipka LJ, LeBeaut AP, Suresh $\mathrm{R}$, et al. Ezetimibe reduces low-density lipoprotein cholesterol: results of a phase III, randomized, double-blind, placebo-controlled trial. Atherosclerosis Suppl. 2001;2(2):38.

83. Ernst ND, Cleeman J, Mullis R, Sooter-Bochenek J, Van Horn L. The National Cholesterol Education Program: implications for dietetic practitioners from the Adult Treatment Panel recommendations. J Am Diet Assoc. 1988;88(11):1401-8.

84. Perk J, De Backer G, Gohlke H, Graham I, Reiner Z, Verschuren $\mathrm{M}$, et al. European Guidelines on cardiovascular disease prevention in clinical practice (version 2012). The Fifth Joint Task Force of the European Society of Cardiology and Other Societies on Cardiovascular Disease Prevention in Clinical Practice (constituted by representatives of nine societies and by invited experts). Eur Heart J. 2012;33(13):1635-701. https://doi. org/10.1093/eurheartj/ehs092.

85. Dai YY, Zhang HS, Zhang XG, Guan QG, Gao Y, Li YZ, et al. Statin-ezetimibe versus statin lipid-lowering therapy in patients with acute coronary syndromes undergoing percutaneous coronary intervention. J Thorac Dis. 2017;9(5):1345-52. https://doi. org/10.21037/jtd.2017.05.46.

86. Tikkanen MJ. Statins: within-group comparisons, statin escape and combination therapy. Curr Opin Lipidol. 1996;7(6):385-8.

87. Huang JC, Lee TY, Liou MJ, Lin CM, Pei D, Chen ZC, et al. Begin with the real-world patients of non-goal-achieved hypercholesterolemia in taiwan through the ezetimibe/simvastatin tablet-the BRAVO Study. Curr Med Res Opin. 2011;27(8):164551. https://doi.org/10.1185/03007995.2011.594425.

88. Yu CC, Lai WT, Shih KC, Lin TH, Lu CH, Lai HJ, et al. Efficacy, safety and tolerability of ongoing statin plus ezetimibe versus doubling the ongoing statin dose in hypercholesterolemic Taiwanese patients: an open-label, randomized clinical trial. BMC Res Notes. 2012;5:251. https://doi. org/10.1186/1756-0500-5-251.

89. Zhang T, Wu WF, Liu Y, Wang QH, Wang LY, Mi SH. Change in cholesterol absorption and synthesis markers in patients with coronary heart disease after combination therapy with simvastatin plus ezetimibe. Chin Med J (Engl). 2013;126(9):1618-23.

90. Rong LV, Li DH, Zhang GD, Zhu A, Yan W. Influences and safety of ezetimibe combining simvastatin on control rate of LDL-C level in patients with coronary heart disease and diabetes. Chin J Evid Based Cardiovasc Med. 2012;4(2):152-4.

91. Cannon CP, Blazing MA, Giugliano RP, McCagg A, White JA, Theroux P, et al. Ezetimibe added to statin therapy after acute coronary syndromes. N Engl J Med. 2015;372(25):2387-97. https://doi.org/10.1056/NEJMoa1410489.

92. Nordestgaard BG, Chapman MJ, Humphries SE, Ginsberg HN, Masana L, Descamps OS, et al. Familial hypercholesterolaemia is underdiagnosed and undertreated in the general population: guidance for clinicians to prevent coronary heart disease: consensus statement of the European Atherosclerosis Society. Eur Heart J. 2013;34(45):3478-90. https://doi.org/10.1093/eurheartj/ eht273.

93. Jarcho JA, Keaney JF. Proof that lower is better - LDL cholesterol and IMPROVE-IT. New Engl J Med. 2015;372(25):2448-50. https://doi.org/10.1056/NEJMe1507041.

94. Lauridsen BK, Stender S, Frikke-Schmidt R, Nordestgaard BG, Tybjaerg-Hansen A. Genetic variation in the cholesterol transporter NPC1L1, ischaemic vascular disease, and gallstone disease. Eur Heart J. 2015;36(25):1601-8. https://doi.org/10.1093/ eurheartj/ehv108.

95. Li L, Zhang M, Su F, Li Y, Shen Y, Shen J, et al. Combination therapy analysis of ezetimibe and statins in Chinese patients with acute coronary syndrome and type 2 diabetes. Lipids Health Dis. 2015;14:10. https://doi.org/10.1186/s12944-015-0004-7.

96. Collins R, Reith C, Emberson J, Armitage J, Baigent C, Blackwell $\mathrm{L}$, et al. Interpretation of the evidence for the efficacy and safety of statin therapy. Lancet. 2016;388(10059):2532-61. https ://doi.org/10.1016/S0140-6736(16)31357-5.

97. Bellosta S, Paoletti R, Corsini A. Safety of statins: focus on clinical pharmacokinetics and drug interactions. Circulation. 2004;109(23 Suppl):III50-7. https://doi.org/10.1161/01. cir.0000131519.15067.1f.

98. Farmer JA, Torre-Amione G. Comparative tolerability of the HMG-CoA reductase inhibitors. Drug Saf. 2000;23(3):197-213.

99. Lee E, Ryan S, Birmingham B, Zalikowski J, March R, Ambrose $\mathrm{H}$, et al. Rosuvastatin pharmacokinetics and pharmacogenetics in white and Asian subjects residing in the same environment. Clin Pharmacol Ther. 2005;78(4):330-41. https://doi.org/10.1016/j. clpt.2005.06.013.

100. Chung N, Cho SY, Choi DH, Zhu JR, Lee K, Lee PY, et al. STATT: a titrate-to-goal study of simvastatin in Asian patients with coronary heart disease. Simvastatin Treats Asians to Target. Clin Ther. 2001;23(6):858-70.

101. Zhu H, Li DY, Xia Y, Xu TD, Ma XL, Zhang YB, et al. Strengthening lipid-lowering effect and safety evaluation on extremely high risk population from ezetimibe therapy combined with simvastatin. Acta Univ Med Nanjing Nat Sci. 2010;30(9):1342-3.

102. Bruckert E, Hayem G, Dejager S, Yau C, Begaud B. Mild to moderate muscular symptoms with high-dosage statin therapy in hyperlipidemic patients-the PRIMO study. Cardiovasc Drugs Ther. 2005;19(6):403-14. https://doi.org/10.1007/s1055 7-005-5686-z.

103. Davidson MH, McGarry T, Bettis R, Melani L, Lipka LJ, LeBeaut AP, et al. Ezetimibe coadministered with simvastatin in patients with primary hypercholesterolemia. J Am Coll Cardiol. 2002;40(12):2125-34. 